\title{
Mejoras en el esquema tecnológico para lograr incrementos en la eficiencia energética en un central azucarero
}

\author{
Improvements in the technological scheme to achieve \\ increases in energy efficiency in a central sugar mill
}

MSc. ReInIER Jiménez Borges (i)

Centro de Estudios de Energía y Medio Ambiente (CEEMA).

Universidad de Cienfuegos Carlos Rafael Rodríguez, Cienfuegos, Cuba.

Ing. Andrés Lorenzo Álvarez González @

Facultad de Ingeniería. Universidad de Cienfuegos Carlos Rafael Rodríguez, Cienfuegos, Cuba.

Ing. Ernesto M. López Alemán (1)

Empresa de Mantenimiento a Centrales Eléctricas (EMCE), Cuba.

MSC. JUNIOR LORENZO LLANES (1)

Departamento de Ingeniería Química y Bioprocesos, Pontificia Universidad Católica de Chile, Santiago, Chile.

Autor para la correspondencia: MSc. Reinier Jiménez Borges

Centro de Estudios de Energía y Medio Ambiente (CEEMA). Universidad de Cienfuegos Carlos Rafael

Rodríguez, Cuatro Caminos Carretera a Rodas, km 3 1⁄2, Cienfuegos, Cuba.

Mail: rei89@nauta.cu; rjborges@ucf.edu.cu

RESUMEN

Este estudio parte de la descripción del esquema térmico del central azucarero a evaluar, el cual cuenta con una molida potencial de 49,67 kg/s, la capacidad eléctrica instalada de 6,5 MW, y con dos generadores de vapor del tipo Retal, los cuales tienen una capacidad nominal de $60 \mathrm{t} / \mathrm{h}$ cada uno, y una presión de vapor sobrecalentado de $1930 \mathrm{kPa}$ a $\left(350^{\circ} \mathrm{C}\right)$. El objetivo fundamental de la investigación fue la realización del balance termo energético de dicho central, con el propósito de conocer los equipos con mayor consumo de vapor y la distribución de la energía en el proceso. Su utilizó una metodología clásica de balance de masa y energía donde a partir del análisis de los principales indicadores globales, siendo ellos el porcentaje de bagazo sobrante, consumo específico de vapor en fábrica, así como electricidad vendida a la red. Al analizar 
dichos indicadores se pudo identificar oportunidades de mejora con el fin de lograr un aumento en la producción eléctrica y entregar mayor energía al Sistema Electroenergético Nacional (SEN), así como disminuir el consumo de vapor de la fábrica. Las modificaciones implementadas luego del análisis fueron la sustitución del turbogenerador del tipo contrapresión de potencia 2,5 MW por uno de 4 MW, así como la concentración de los jugos. Mediante la implementación de estas propuestas se logró un incremento en la entrega de electricidad al SEN de 8,88 kWh/ton de caña para una reducción del consumo de vapor en la fábrica de 25,56 \%. Finalmente, se compara desde el punto de vista económico el incremento en los ingresos para el central, lográndose para este caso un excedente de 35008060.9 USD/zafra.

Palabras claves: Cogeneración, Eficiencia, Esquema, Simulación, Indicadores, Vapor

\section{ABSTRACT}

The current study starts from the description of the thermal scheme of the sugar plant to be evaluated. This scheme basically has a ground potential of $49.67 \mathrm{~kg} / \mathrm{s}$, the installed electrical capacity of 6,5 MW, and two Retal-type steam generators which have a nominal capacity of $60 \mathrm{t} / \mathrm{h}$ each, and a superheated vapor pressure of $1930 \mathrm{kPa}$ at $\left(350^{\circ} \mathrm{C}\right)$. The fundamental objective of the research was to carry out the thermoenergetic balance of said plant, in order to know the equipment with the highest steam consumption and the energy distribution in the process. For this, a classical methodology of mass and energy balance was selected where, based on the analysis of the main global indicators, being the $\%$ of excess bagasse, specific consumption of steam in the factory, as well as electricity sold to the network, they allowed identifying opportunities for improvements in order to achieve an increase in electricity production and deliver more energy to the National Electroenergetic System (SEN), as well as reduce the steam consumption of the factory. These modifications were in the replacement of the 2,5 MW back pressure type turbogenerator with one of $4 \mathrm{MW}$, as well as the concentration of the juices. Through the implementation of these proposals, an increase in the delivery of electricity to the SEN of 8,88 kWh/ton of sugarcane was achieved for a reduction of steam consumption in the factory of $25,56 \%$. Finally, from the economic point of view, the increase in income for the plant is compared, achieving in this case a surplus of 35,008,060.9 USD/harvest.

Keywords: Cogeneration, Efficiency, Scheme, Simulation, Indicators, Steam

\section{Introducción}

Históricamente la economía cubana ha estado sustentada por la industria azucarera llegando a ser en años anteriores uno de los mayores productores de este cristal. La diversificación de la Industria Azucarera planteada por Cuba aprovecha el potencial que ofrece la disponibilidad de biomasa cañera y el desarrollo de las tecnologías de cogeneración (Pérez 2017).

La industria azucarera es privilegiada porque de la materia prima que procesa se obtiene - además del azúcar - el agua y el combustible necesarios para su operación. El bagazo de caña, biomasa residual del proceso de extracción del jugo, posee un excelente valor calórico de 7,64 $\mathrm{MJ} / \mathrm{kg}$ para un $50 \%$ de humedad, similar al poder calórico de la madera 7,9 MJ/kg (Hernán-
dez-Sardiñas 2017), y constituye un combustible renovable que incinerado en los generadores de vapor produce el vapor que mueve las turbinas de contrapresión para el accionamiento de los generadores eléctricos (Jiménez, 2016).

Actualmente, la industria azucarera aporta el 3,5 por ciento de la generación de electricidad del Sistema Electroenergético Nacional (SEN) de Cuba. El plan para elevar la eficiencia energética en el país contempla que para 2030 una veintena de proyectos generen un excedente de 755 MW que pueden ser suministrados a la red del SEN. Actualmente, las fuentes de energías renovables solo representan el 4,6 por ciento de la generación eléctrica y el resto lo aportan los combustibles fósiles. La paulatina instalación en los centrales azucareros de modernas plantas bioeléctricas, necesarias para lograr ese objetivo, 
requiere una inversión, que la empresa azucarera espera obtener de préstamos gubernamentales o inversiones extranjeras (Alemán 2017).

La industria azucarera en Cuba se encuentra atravesando un proceso de reordenamiento y redimensionamiento con el objetivo de alcanzar mayores beneficios tecnológicos, económicos y ecológicos, impuestos por las condiciones económicas y medioambientales en que se desarrolla actualmente. En Cuba existe actualmente una capacidad instalada de 470 MW en 57 centrales azucareros del país. Como proyección para el año 2030 se estima el empleo de 25 bioeléctricas con una capacidad de 950 MW (Jiménez 2016).

No obstante, las insuficiencias que aún prevalecen en los sistemas de gestión energética en el sector azucarero que han sido detectadas por diversos autores, grupos e instituciones en el sector empresarial en Cuba, la inestabilidad en el aprovechamiento de la capacidad instalada que a su vez depende en zafra de la norma potencial de molida y en el periodo inactivo de la operación de la planta eléctrica y la obsolescencia tecnológica de la base energética de la industria, entre otros factores, conllevan a la elevación de los consumos y costos energéticos, y al no aprovechamiento de las potencialidades para la generación y entrega de electricidad al SEN (Bernal 2016).

Para llegar a conocer las condiciones actuales de los esquemas de las fábricas de azúcar es necesario la aplicación de un balance termo energético integral, pues este puede señalar las dificultades existentes y conducir a mejoras económicas, evitándose el consumo de combustible adicional, o en caso de sobrante estudiar las formas económicamente más ventajosas de su utilización, por lo que constituye el objetivo fundamental del estudio. La realización de un balance termo energético en una fábrica de azúcar es una tarea compleja, por el gran número de variables y equipos consumidores de vapor que intervienen en el proceso de elaboración (Falcón et al. 2019).

\section{Materiales y métodos}

El esquema de uso del vapor es esencial a la hora de analizar todo el vapor generado y la eficiencia del sistema necesaria para generar electricidad de todo el proceso de fabricación de azúcar en el central y para la venta a la red pública.

En la Figura 1 se presenta el esquema energético de un central azucarero tradicional, caracterizado por la simplicidad de su esquema. La presión del vapor directo es baja al igual que lo es la presión de escape. Como corresponde a un esquema elemental de consumo, los calentadores de jugo, el cuádruple efecto y los tachos reciben vapor de escape. Este esquema dimensionado para una capacidad base de $100000 @$ (1 150 tc/d)contaba con una presión de trabajo del generador de vapor de $1030 \mathrm{kPa}\left(10.5 \mathrm{~kg} / \mathrm{cm}^{2}\right)$ y una temperatura del vapor de $206^{\circ} \mathrm{C}$ (Díaz, 2016). El esquema presentado sirve para comparar la evolución de los esquemas tecnológicos cubanos, así como los parámetros del vapor y características del proceso. La mayor parte de los esquemas azucareros actuales operan con generadores de vapor de media presión, turbogeneradores de vapor del tipo contrapresión con capacidades entre $4 \mathrm{MW}$ y $8 \mathrm{MW}$ por citar algunas de las características fundamentales. 
Figura 1.

Esquema térmico tradicional para una capacidad base de 100000 @. Fuente: Díaz 2016.

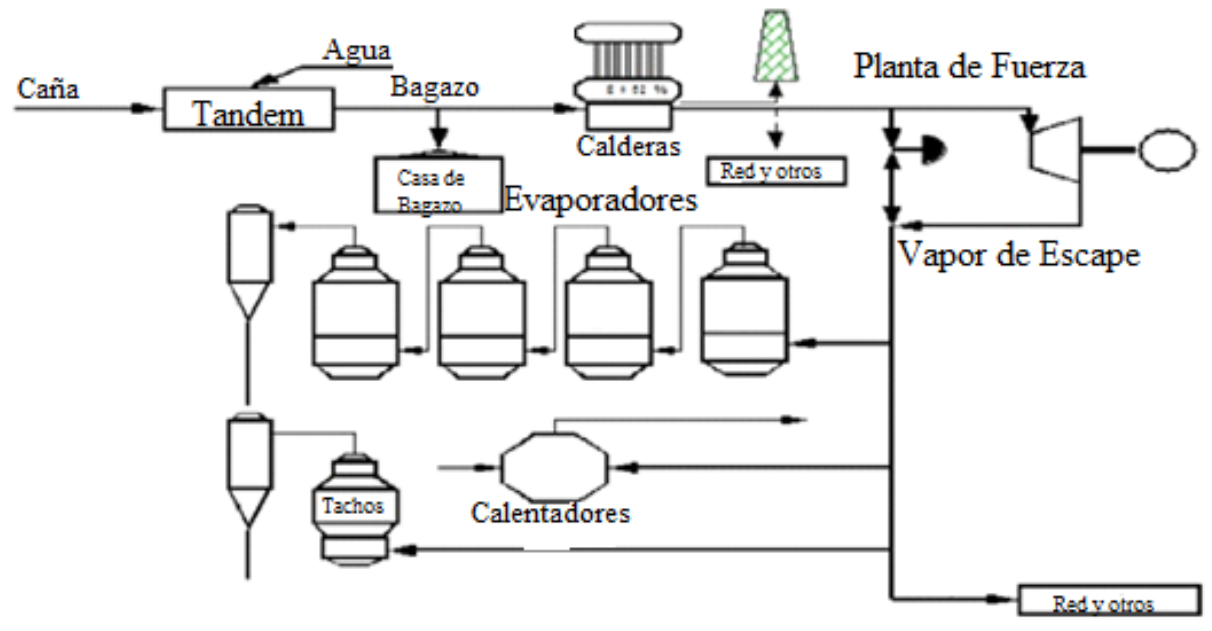

El central azucarero objeto de estudio, con una molida potencial de 49,67 kg/s (4 $300 \mathrm{t} / \mathrm{d}) \mathrm{de}$ caña, produce azúcar crudo. El tándem es accionado eléctricamente, la alcalización se realiza en frío y el sistema de cocción es de tres masas cocidas. La fábrica tiene una capacidad eléctrica instalada equivalente a 6,5 MW. Cuenta con una batería de cinco molinos de modelo Fulton con virgen inclinada en el área de molienda. El sistema de generación de vapor del central cuenta con dos calderas del tipo Retal los que tienen una capacidad nominal de $60 \mathrm{t} / \mathrm{h}$, una presión de vapor sobrecalentado de $1930 \mathrm{kPa}$ (180 lbf/ $\left.\mathrm{in}^{2}\right)$ a una temperatura de $623,15 \mathrm{~K}\left(350^{\circ} \mathrm{C}\right)$. En el área de evaporación se disponen con calentadores líquido a líquido, vampiros y WEBER modificados y un clarificador BTR, que calientan el jugo a temperaturas desde $375,15 \mathrm{~K}$ a $378,15 \mathrm{~K}$ $\left(102-105^{\circ} \mathrm{C}\right)$ y este es enviado a los pre-evaporadores. El esquema de evaporación está formado por un quíntuple efecto, con dos primeros vasos consumidores de vapor de escape en paralelo y con el jugo en serie, el mismo suministra vapor a los tachos, a los calentadores secundario y rectificador de jugo alcalizado, estos operan a presiones relativamente altas $124 \mathrm{kPa}$ a $103 \mathrm{kPa}$ ó (18 a 15 lbf/in²). En la estación de tachos están colocados un total de siete tachos, además en esta área se cuenta con un cristalizador rápido de 3ra, dos graneros, dos cristalizadores tradicionales de azúcar comercial y finalmente posee 4 centrifugas comerciales y otras 5 polacas para masa cocida B y tres centrifugas de doble semilla. En la Figura 2 se presenta el diagrama de flujo de proceso para el caso base de este central.

Para la evaluación termoenergética del central se utilizó una hoja de cálculo Excel y se realizó primeramente el balance de materia en el área de generación de vapor para determinar las corrientes que intervienen en las operaciones involucradas, y luego se procede con el balance energético para conocer los equipos con mayor consumo de vapor y la distribución de la energía en el proceso, para ello se utilizó la metodología propuesta por Espinoza (1991) y Pérez (2017). 
Figura 2.

Diagrama de flujo de proceso para el central objeto de estudio. Fuente: elaboración propia.

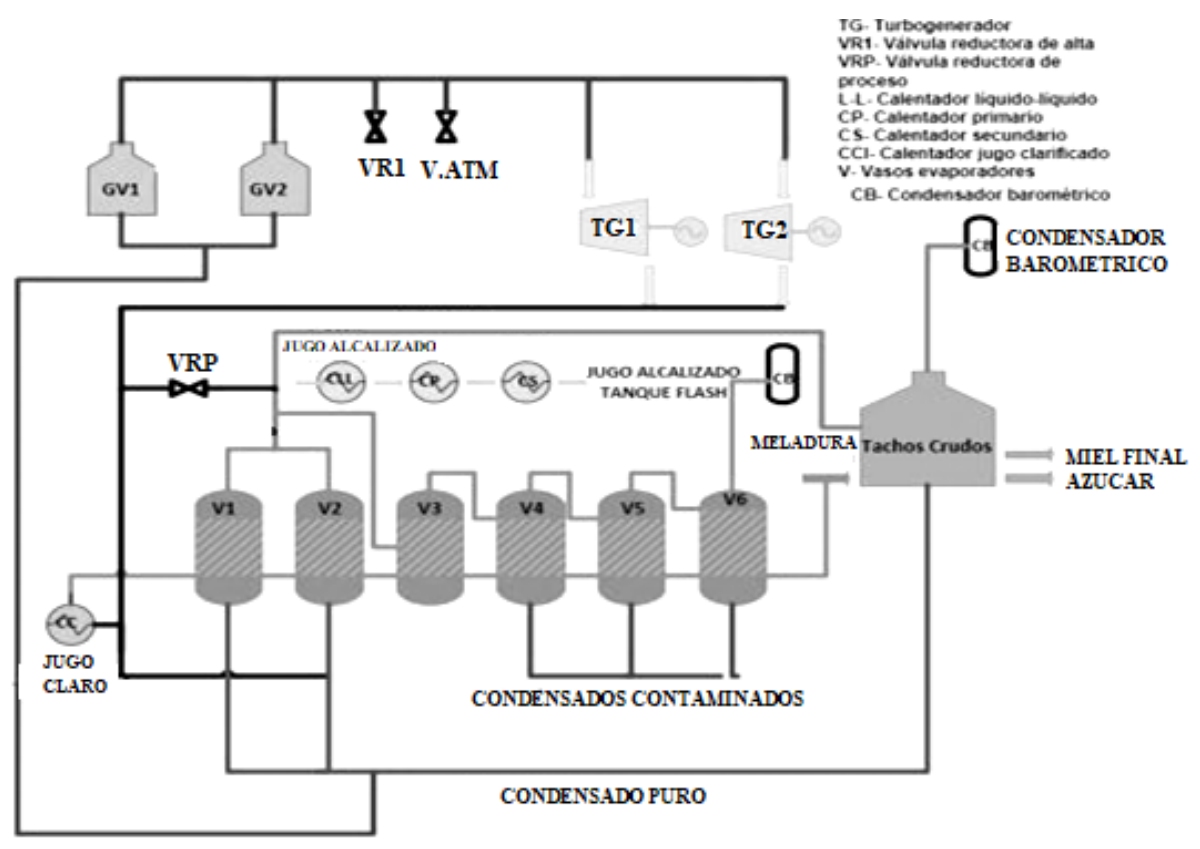

El balance de materiales se realizó mediante los datos brindados por la fábrica y las ecuaciones para el balance total de masa en las etapas de extracción-alcalización-clarificación. Las figuras 3-5 representan las etapas de cada uno de los procesos para la realización de los balances, donde se cuenta con la información inicial de cada una de las corrientes (composición de sacarosa, $\%$ sólidos solubles, \% de agua). Las ecuaciones 1-6 representan estas etapas.

Para el caso de la etapa de extracción- alcalización-clarificación se pueden plantear tres ecuaciones linealmente independientes, donde se presentan a partir de las Ec.1, Ec.5, Ec.6 las correspondientes al balance global.

\section{Etapa de extracción}

\begin{tabular}{ll}
\hline $\mathrm{CM}+\mathrm{A}=\mathrm{MB}+\mathrm{JM}$ & Ec. 1 \\
$\mathrm{MB}=\mathrm{B} * \mathrm{CM} / 100$ & Ec. 2 \\
$\mathrm{JM}=\mathrm{CM}{ }^{*} \mathrm{Em}$ & Ec. 3 \\
$\mathrm{~m}($ lechada $)=\mathrm{V}($ lechada $) * \rho($ lechada $)$ & Ec. 4 \\
$\mathrm{JM}+\mathrm{JF}+\mathrm{LC}=\mathrm{JA}$ & Ec. 5 \\
\hline
\end{tabular}


Figura 3.

Etapa de extracción.

Agua de imbibición

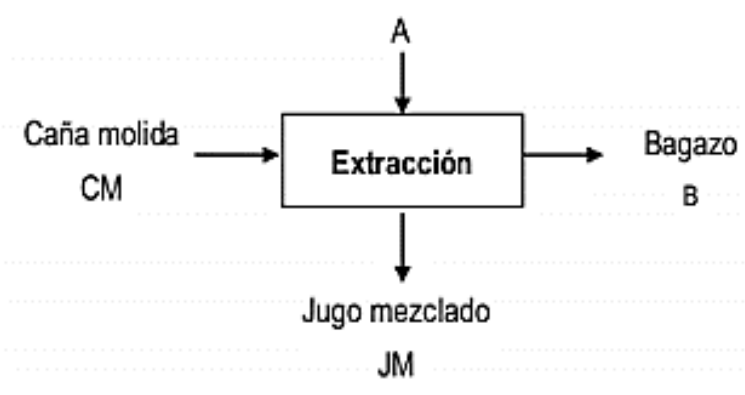

Fuente: Elaboración propia.

Figura 4.

Etapa de alcalización.

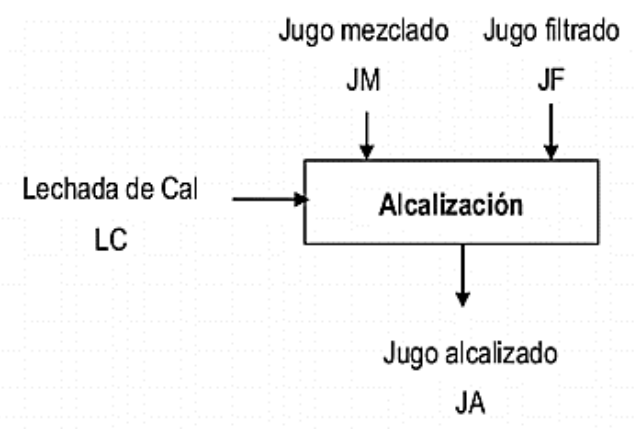

Fuente: Elaboración propia.

\section{Etapa de clarificación}

$$
\begin{array}{ll}
\mathrm{JA}+\mathrm{F}=\mathrm{JC}+\mathrm{C} & \text { Ec. } 6
\end{array}
$$

Figura 5.

Etapa de clarificación.

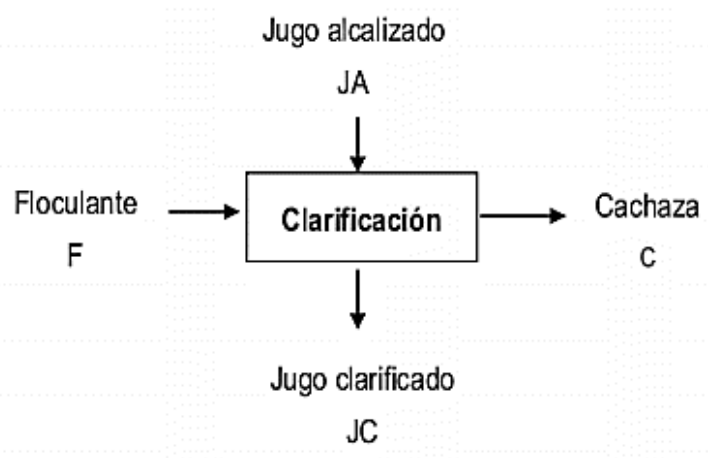

En la realización del balance energía, fue incluido el balance de vapor, de condensados y de calor en los equipos del esquema termo energético. Las ecuaciones utilizadas en los balances de vapor, generadores de vapor y condensados fueron las ecuaciones 7-16.

$\begin{array}{ll}\text { Turbogeneradores } \\ \mathrm{GMP}=\frac{860 * \mathrm{Ntg}}{\Delta \mathrm{H} * \eta_{\mathrm{t}} * \eta_{\text {elec }} * \eta_{\text {mec }} * \eta_{\text {tubo }}} & \text { Ec. } 7\end{array}$

Calentador líquido-líquido.

$\begin{array}{ll}\mathrm{Gl}-\mathrm{l}=\frac{\mathrm{JMF} * \mathrm{Cp} *(\mathrm{Ts}-\mathrm{Te})}{\mathrm{C}_{\mathrm{pH}_{2} \mathrm{O}} *(\mathrm{Te}-\mathrm{Ts})} * \mathrm{X} & \text { Ec. } 81 \\ \mathrm{Cp}=1-0,0056 * \mathrm{Bx} & \text { Ec. } 91\end{array}$

Calentadores primarios y rectificadores.

$$
\mathrm{Gc}=\frac{\mathrm{JMF} * \mathrm{Cp} *(\mathrm{Ts}-\mathrm{Te})}{\lambda \mathrm{v}} * \mathrm{X} \quad \text { Ec. } 10
$$

Calentador de jugo clarificado.

$\mathrm{Gc}=\frac{\mathrm{JC} * \mathrm{Cp} *(\mathrm{Ts}-\mathrm{Te})}{\lambda \mathrm{v}} * \mathrm{X} \quad$ Ec. 11

Balance parcial en el ler preevaporador

$$
\mathrm{Js}_{1 \text { pre }}=\mathrm{JC} * \mathrm{Bx}_{\mathrm{JC}} / \mathrm{Bx}_{\text {salida } 1 \text { pre }} \quad \text { Ec. } 12
$$

Balance parcial en el 2do preevaporador.

$$
\mathrm{Js}_{2 \text { pre }}=\mathrm{Js}_{1 \text { pre }} * \mathrm{Bx}_{\text {salida 1pre }} / \mathrm{Bx}_{\text {salida 2pre }} \text { Ec. } 13
$$

Vapor producido $(\mathrm{kg} / \mathrm{h})$

$$
\text { Gpre }=\left(\frac{\mathrm{JC} * \mathrm{Cp} *(\mathrm{Teb}-\text { Talim })}{\lambda v}+\frac{\text { Vproducido } * \lambda \mathrm{c}}{\lambda \mathrm{v}}\right) \mathrm{X} \quad \text { Ec. } 14
$$

Cuádruple efecto.

$$
\mathrm{G} 1 \mathrm{v}=\left(\frac{\mathrm{Js} 2 \mathrm{pre} * \mathrm{Cp} *(\mathrm{Teb}-\text { Talim })}{\lambda \mathrm{v}}+\frac{\lambda c}{\lambda \mathrm{c}}+\frac{\mathrm{W}}{\mathrm{n}}\right) * \mathrm{x} \quad \text { Ec. } 15
$$

Tachos.

$\mathrm{Gtacho}=\frac{w 1+w 2}{t} * 1,1$

Ec. 16

Fuente: Elaboración propia. 
Para la realización del análisis económico se tomaron en cuenta las corrientes de ingresos y egresos para el central, estos datos aportados por el departamento económico permiten estimar las ganancias por concepto de venta de electricidad al SEN y por venta de azúcar, siendo las de mayor contribución dentro del esquema evaluado. La Figura 3 presenta los egresos e ingresos por compra y venta de productos en un central, en este caso particular el excedente de bagazo sobrante, así como el petróleo que pudiera ser consumido en algún momento de zafra no se venden o compran en el caso evaluado. Los precios de venta o compra de las restantes corrientes fueron consideradas en el análisis para ilustrar las posibles ganancias netas a partir de la evaluación del caso mejorado con respecto al caso base y forman parte de información restringida para el uso.

\section{Resultados y discusión}

En la Tabla I se presentan los resultados obtenidos por las ecuaciones de la metodología seleccionada, del consumo de vapor de los equipos que conforman el proceso de fabricación del azúcar.

Tabla I.

Resultados obtenidos en el balance de vapor.

\begin{tabular}{lr}
\hline Equipos & Valor del consumo $(\mathrm{kg} / \mathrm{h})$ \\
\hline Total en turbogeneradores & 74968,10 \\
Total en calentadores & 35649,88 \\
ler vaso del cuádruple efecto & 25369,02 \\
Pre-evaporador & 69021,73 \\
Total en tachos & 73258,69 \\
Equipos tecnológicos & 79121,09 \\
Otros consumos & 7131,73 \\
Generadores de vapor & 88481,66 \\
\hline
\end{tabular}

\section{Análisis de los indicadores del caso base}

Mediante los resultados obtenidos se analiza el comportamiento general del central en cuanto a indicadores reportados por la literatura. Los indicadores operacionales o de comportamiento del STE son elementos que pueden servir de guía para facilitar la fase de análisis tanto desde el punto de vista integral (indicadores globales) como los de áreas y equipos. Estos valores deben usarse como reglas que, - adecuadamente empleadas - permiten obtener una idea primaria de los posibles problemas, deficiencias, limitaciones y/o aspectos positivos. En las Tablas II y III se muestran los indicadores globales y por áreas que se obtienen como resultado del balance realizado. 
Tabla II.

Indicadores globales del central evaluado.

\begin{tabular}{lrr}
\hline Indicadores globales & Valor & Unidad \\
\hline \% Bagazo sobrante & 7,56 & $\%$ \\
Bagazo disponible \% de caña & 36 & $\%$ \\
Consumo vapor de la fábrica \% caña & 44,16 & $\%$ \\
Demanda eléctrica especifica de la fábrica & 32 & $\mathrm{~kW}-\mathrm{h} /$ ton de caña \\
Producción eléctrica específica de la fábrica & 37,03 & $\mathrm{~kW}-\mathrm{h} /$ ton de caña \\
Producción eléctrica entregada al SEN & 5,02 & $\mathrm{~kW}-\mathrm{h} /$ ton de caña \\
\hline
\end{tabular}

Tabla III.

Indicadores por área.

\begin{tabular}{lrr}
\hline Indicadores por área & Valor & Unidad \\
\hline Brix jugo claro & 14,41 & $\%$ \\
Brix meladura & 64,28 & $\%$ \\
Economía de área de evaporación & 4,06 & - \\
\hline
\end{tabular}

El bagazo sobrante según Victoria et al. (2009), en ingenios azucareros cubanos se comporta entre un 5 a un $30 \%$ mientras que (Rein 2012) recomienda entre un 5 y $10 \%$. Motito (2014) obtuvo en la evaluación energética de un central un sobrante de bagazo de un 5,74\%, mientras que Hernández et al (2020) evalúan la gestión energética en un central de azúcar crudo cuyo indicador de bagazo sobrante fue de un 25,23 \%. Por otro lado, Hernández-Sardiñas (2017) en el resumen de los balances de masa y energía obtiene un $5,49 \%$ de bagazo sobrante, mientras que Jiménez-Borges (2017) en su estudio presenta un sobrante de bagazo de 33,98\%. En la simulación de un sistema termo energético estimó el sobrante de bagazo en 20,68\%. Para garantizar el combustible necesario para las paradas por falta de caña o interrupciones operacionales del ingenio, o el arranque del mismo en la zafra siguiente. Por eso este indicador no puede ser cero, esto traería consigo la implementación de otro combustible, lo que es económicamente deficiente. En el caso de estudio el sobrante de bagazo que se obtuvo fue de un 7,56\%. Lo que le permite al central el arranque en caso de parada imprevista o mantenimiento.

El bagazo disponible \% de caña según Rein (2012) puede estar entre 22 y $35 \%$ de caña, pero es más común un valor entre un 25 a $30 \%$ mientas que Victoria et al. (2009) expresa un intervalo de un $27 \%$ a un $30 \%$ estos valores son necesarios para garantizar una producción de vapor en base continua. La cantidad de bagazo disponible obtenida en el central es de un $36 \%$ por lo que es capaz de satisfacer la cantidad de vapor necesaria de forma continua. 


\section{El vapor consumido directo a la fábrica de-} pende de la cantidad de electricidad generada. También de la estabilidad en el consumo promedio de vapor de baja presión en los tachos; y de la potencia instalada de los turbogeneradores pues los equipos de mayor potencia tienen menor consumo específico y de si se expulsa vapor a la atmósfera. En el proyecto el consumo de vapor está en un $44,16 \%$ que se encuentra entre el intervalo que hace referencia la bibliografía que es de un 35 a un $48 \%$ según Victoria et al. (2009) y de un 40 a un 60 \% según Rein (2012), Hernández et al. (2020) determina un consumo de vapor en fábrica para el esquema termo energético evaluado de 43,61\%. Iturralde (2020) determinó en 44, 73\% el consumo de vapor en fábrica a partir de una propuesta metodológica para un balance termo energético en un central azucarero, mientras que este indicador según Jiménez-Borges (2017) en el estudio propuestas para incrementar la entrega de electricidad en un central azucarero fue de $40,52 \%$. Previamente en la simulación de un esquema termo energético se estimó un consumo de vapor en fábrica de $46,47 \%$.

La demanda eléctrica especifica de la fábrica es el consumo eléctrico que tiene la fábrica para el proceso de fabricación del azúcar, este está dado principalmente por los motores eléctricos instalados en el área de molienda y otra parte por las oficinas. La fábrica presenta una demanda eléctrica de $32 \mathrm{~kW}$-h/ton de caña este valor debe de estar en el intervalo de un 15 a $30 \mathrm{~kW}-\mathrm{h} /$ ton de caña en la industria azucarera cubana (Victoria et al. 2009) aunque Rein (2012) refleja que puede llegar hasta los $35 \mathrm{~kW}$-h/ton de caña. La demanda eléctrica específica en fábrica según Jiménez-Borges (2017) fue de 31,78 kW-h/ ton caña para el central azucarero evaluado en el estudio.
La producción eléctrica específica de la fábrica es toda la energía eléctrica que es capaz de producir la fábrica a partir de la cogeneración, esta depende de los turbogeneradores y de la capacidad eléctrica instalada. El central tiene una capacidad instalada de $6500 \mathrm{~kW}$, aunque solo se genera $4900 \mathrm{~kW}$. Tiene una producción eléctrica en fábrica de 37,03 kW-h/ton de caña.

\section{La energía eléctrica entregada al SEN esta} depende de la demanda eléctrica de la fábrica y la capacidad instalada del central azucarero. Esta se puede aumentar si se ponen a trabajar lo turbogeneradores a la capacidad nominal. En nuestro caso se entrega al SEN 5,03 kW-h/ton de caña.

Brix jugo claro. La concentración del jugo clarificado debe estar entre los intervalos de 15 a 17 \% según Victoria et al. (2009) mientras que el caso base analizado es de $14,41 \%$, por lo que se necesita más consumo de vapor entre otras razones para poder calentar el jugo y aumentar su concentración.

Brix meladura, la meladura es el resultado de la salida de la estación de evaporación, es la mezcla que entra en la estación de tachos para el proceso de cristalización, este debe estar entre un rango de 64 a $67 \%$ de concentración. El valor que alcanza el central es de 64,28 \%, un valor próximo al límite inferior por lo que genera un mayor consumo de vapor en la estación de tachos. Jiménez-Borges (2017) considera por su parte un Brix de la meladura en 63,8\%.

\section{Identificación de oportunidades de mejoras}

Para mejorar la eficiencia y ahorrar combustible en la industria azucarera existen diversas vías, entre las cuales se pueden mencionar el estudio 
del sistema actual para la introducción de mejoras que determinen una mayor eficiencia del sistema energético, los cambios para alcanzar una mayor utilización del vapor con vistas a la optimización y la sustitución de equipos de baja eficiencia térmica por otros de mayor eficiencia.

Por ello, a partir de la aplicación del balance energético, se proponen modificaciones al esquema térmico, evaluando su factibilidad técnica y económica para el proceso de producción de azúcar crudo con la aplicación de la metodología propuesta a partir del cálculo analítico.

Las principales modificaciones a tener en cuenta para el esquema termo energético actual son:

\section{Sustitución del turbogenerador del tipo contrapresión de potencia 2,5 MW por uno de $4 \mathrm{MW}$}

Actualmente el central cuenta con una potencia total instalada de 6,5 MW teniendo un turbo de 4 MW y otro de 2,5 MW. Para ello se plantea incrementar su potencia instalada sustituyendo el turbogenerador de 2,5 MW por uno de $4 \mathrm{MW}$ existente en el central, con el objetivo principal de entregar mayor energía eléctrica al SEN sin afectar el proceso de producción de azúcar por zafra del central.

\section{Concentración de los jugos ${ }^{0}$ Brix}

Aunque la concentración de los jugos está cercana al intervalo recomendado esto no significa que no se pueda interactuar con ellos. Se regularon los ${ }^{0}$ Brix en los dos primeros vasos para lograr el compromiso entre la disponibilidad de vapor producido por ellos y la demanda de los calentadores y tachos. Con el fin de evitar la expulsión de vapor a la atmosfera.

A partir de la evaluación de un caso mejorado incorporando estas oportunidades detectadas se obtuvieron resultados positivos en el aumento de algunos de los indicadores globales para este caso en comparación con el base. Los valores modificados, así como los resultados obtenidos se pueden apreciar en las Tabla IV.

\section{Tabla IV.}

Valores modificados y resultados obtenidos en los casos analizados.

\begin{tabular}{|c|c|c|c|c|}
\hline Datos & Unidad & Caso base & Caso mejorado & Diferencia \\
\hline 0Brix salida ler pre-evaporador & $\%$ & 18,4 & 16,4 & 2,0 \\
\hline 0Brix salida 2 do pre-evaporador & $\%$ & 24,1 & 19,8 & 4,3 \\
\hline 0Brix Jugo Claro & $\%$ & 14,4 & 17 & 3,4 \\
\hline 0Brix Meladura & $\%$ & 64,3 & 67,0 & 3,3 \\
\hline Turbogenerador de contrapresión & $\mathrm{kW}$ & 2500 & 4000 & 1500 \\
\hline Potencia total & $\mathrm{kW}$ & 6500 & 8000 & 1500 \\
\hline \multicolumn{5}{|c|}{ Indicador } \\
\hline Consumo de vapor de la fabrica & $\%$ & 44,2 & 18,6 & 25,6 \\
\hline Producción eléctrica de la fabrica & $\mathrm{kWh} /$ ton de caña & 37,0 & 45,9 & 8,9 \\
\hline Entrega al SEN & $\mathrm{kWh} /$ ton de caña & 5,0 & 13,9 & 8,9 \\
\hline
\end{tabular}




\section{Análisis económico}

Los principios que se presentan a continuación son válidos para cualquier tipo de proceso, pero para este caso se reducen a los aspectos vinculados solamente a la producción de azúcar cru- do. En el diagrama de la Figura 6 se muestran los egresos e ingresos que se tienen en cuenta en la determinación de las ganancias netas, lo que debe servir de guía, tanto para realizar el análisis económico como para orientarse en la búsqueda de alternativas.

Figura 6.

Egresos e ingresos por compra y venta de productos en ingenio de crudo.

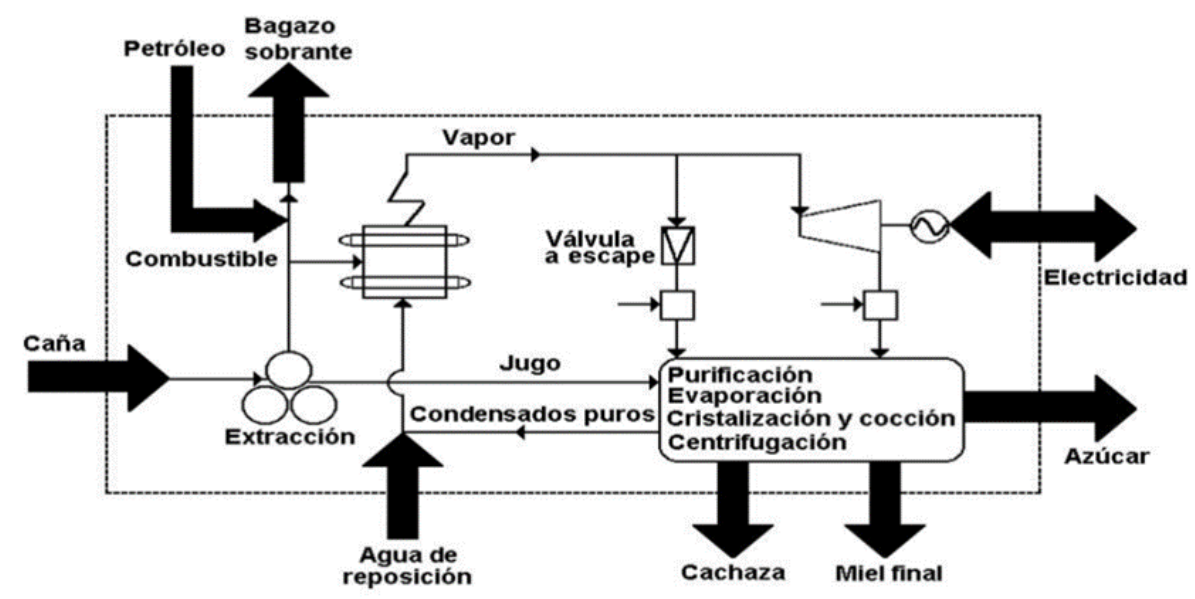

Fuente: Victoria et al. 2009.

A partir de la metodología utilizada por Victoria et al. (2009) para el cálculo económico se realizó un análisis del caso base con el caso mejorado mediante los datos proporcionados por el cen- tral. En la Tabla V se muestran los ingresos y egresos de cada una de las variables de producción y consumo del central azucarero.

Tabla V.

Ingresos y egresos de los casos analizados.

\begin{tabular}{|c|c|c|c|}
\hline Ingresos & Caso base & Caso mejorado & Unidad \\
\hline Ingreso por concepto de electricidad vendida a la red & 1.998 .057 & 5.523 .398 & USD \\
\hline Ingreso por concepto de venta de azúcar & 201.850 .560 & 233.242 .560 & USD \\
\hline Ingreso por concepto de venta de miel & 531.360 & 622.080 & USD \\
\hline Ingreso por concepto de venta de cachaza & 115.200 & 115.200 & USD \\
\hline Total & 204.495 .177 & 239.503 .238 & USD \\
\hline \multicolumn{4}{|l|}{ Egresos } \\
\hline Costo por concepto de caña comprada & 90.301 .680 & 90.301 .680 & USD \\
\hline
\end{tabular}


Mediante los resultados obtenidos sobre los ingresos y egresos el central para el caso base se obtienen ganancias con un valor de 114.193.497 USD/zafra mientras que para el caso mejorado las ganancias obtenidas son de 149.201.558 USD/zafra.

\section{Conclusiones}

Los principales indicadores globales obtenidos a través del balance térmico fueron: El bagazo sobrante con un valor de 7,56\% y a su vez el bagazo disponible con un $36 \%$, el consumo de vapor de la fábrica determinado fue de un $44,16 \%$. Se obtuvo una demanda eléctrica especifica de fábrica de $32 \mathrm{kWh} /$ ton de caña, la producción eléctrica específica de 37,03 kWh/ton de caña entregándose al SEN 5,02 kWh/ton de caña.

Las principales oportunidades detectadas para aumentar la eficiencia a partir del análisis del caso base fueron: aumentar la capacidad potencial del central, sustitución del turbogenerador de contrapresión de 2,5 MW de potencia y el aumento de la concentración del jugo claro y la meladura, así como disminuir la concentración a la salida de los pre-evaporadores.

Las propuestas de modificaciones al esquema térmico favorecieron el aumento de indicadores de eficiencia como: la producción eléctrica hasta 45,90 kWh/ton de caña, la entrega al SEN de 13,9 kWh/ton de caña y la disminución del consumo de vapor en fábrica hasta un 18,60 \% .

\section{Nomenclatura}

SEN: Sistema Electroenergético Nacional

CM: Cantidad de caña molida que entra a los molinos $(\%)$
A: Agua de imbibición suministrada a los moli$\operatorname{nos}(\%)$

MB: Masa de bagazo resultante del proceso de molienda $(\%)$

B: Por ciento de bagazo en caña $(\%)$

JM: Jugo mezclado o la masa de jugo en los mo$\operatorname{linos}(\%)$

Em: Por ciento de extracción en los molinos (\%) $\rho$ (lechada): Densidad de Oxido de Calcio $(\mathrm{CaO})$ $(\mathrm{kg} / \mathrm{L})$

V(lechada): Volumen de la lechada de cal (L)

Jugo filtrado a partir del jugo mezclado en el proceso de molienda $(\%)$

LC: Lechada de cal (\%)

JA: Jugo alcalizado (\%)

Floculante $(\%)$

C: Cachaza (\%)

JC: Jugo clarificado (\%)

GMP: Consumo de vapor específico del turbogenerador $(\mathrm{kg} / \mathrm{h})$

$\Delta \mathrm{H}$ : Diferencia de entalpía entre el vapor a la entrada de la turbina y después de la expansión ideal $(\mathrm{kcal} / \mathrm{kg})$

860: Conversión de kWh a kcal

Ntg Potencia real del turbogenerador $(\mathrm{kW})$

$\eta_{\text {elec }}$ : Eficiencia eléctrica: $0,90 \div 0,94$

пmec: Eficiencia mecánica: 0,98

ұtub : Eficiencia de la tubería.

$\eta_{\mathrm{t}}$ Eficiencia termodinámica

Gl-1: Consumo de vapor en el calentador liquido-liquido $(\mathrm{kg} / \mathrm{h})$

JMF: Flujo de guarapo a licor $(\mathrm{JM}+\mathrm{JF})(\mathrm{kg} / \mathrm{h})$

Cp: Calor específico del jugo $\left(\mathrm{kcal} / \mathrm{kg}^{\circ} \mathrm{C}\right)$

$\mathrm{C}_{\mathrm{pH} 2 \mathrm{O}}$ : Calor específico del agua $\left(\mathrm{kcal} / \mathrm{kg}^{\circ} \mathrm{C}\right)$

Ts: Temperatura de salida del jugo $\left({ }^{\circ} \mathrm{C}\right)$

Te: Temperatura de entrada del jugo $\left({ }^{\circ} \mathrm{C}\right)$ 
X: Factor de pérdidas, (1,02-1,04)

Gc: Consumo de vapor de los calentadores primarios y rectificadores $(\mathrm{kg} / \mathrm{h})$

$\lambda v$ : Calor latente del vapor utilizado $(\mathrm{kcal} / \mathrm{kg})$

$\mathrm{Js}_{1 \text { pre }}$ : Jugo a la salida del primer pre-evaporador $(\mathrm{kg} / \mathrm{h})$

$\mathrm{Bx}_{\mathrm{JC}}$ : Concentración del jugo clarificado (\%)

Bxsalida $_{1 \text { pre }}$ : Concentración del jugo a la salida del ler pre-evaporador $(\%)$

$\mathrm{Js}_{1 \text { pre }}:$ Jugo a la salida del segundo pre-evaporador $(\mathrm{kg} / \mathrm{h})$

$\mathrm{Bx}_{\text {salidalpre }}$ : Concentración del jugo a la salida del 2do pre-evaporador $(\%)$

G1v: Consumo de vapor en el primer vaso $(\mathrm{kg} / \mathrm{h})$

W: Productividad total $(\mathrm{kg} / \mathrm{h})$

n: número de efectos

$\mathrm{Bx}_{\text {concent.1 }}$ : Concentración a la entrada del múltiple $(\%)$

$\mathrm{Bx}_{\text {meladura }}$ : Concentración a la salida del múltiple $(\%)$

$\lambda c$ : Calor latente de vapor del ler efecto, en el cuerpo $(\mathrm{kcal} / \mathrm{kg})$

$\lambda v$ : Calor latente de vapor del primer efecto, en la calandria $(\mathrm{kcal} / \mathrm{kg})$

Cp: Calor específico del jugo de entrada, (kcal/ $\operatorname{kg}^{\circ} \mathrm{C}$ )

$\mathrm{T}_{\mathrm{eb}}$ : Temperatura de ebullición del primer cuerpo $\left({ }^{\circ} \mathrm{C}\right)$

Ta: Temperatura de la alimentación $\left({ }^{\circ} \mathrm{C}\right)$

Gc. primarios: Consumo de vapor de calentadores primarios $(\mathrm{kg} / \mathrm{h})$

X: Coeficiente de perdida 1,04

E: Economía

$\mathrm{G}_{\text {tacho }}$ : Consumo de vapor en el tacho $(\mathrm{kg} / \mathrm{h})$

$\mathrm{V}_{\text {final }}$ : Volumen final de la templa (m3)

$\mathrm{V}_{\text {inicial }}:$ Volumen inicial del tacho (m3) $\gamma$ : Peso específico del material. Se puede tomar como $1450\left(\mathrm{~kg} / \mathrm{m}^{3}\right)$ valor promedio de acuerdo con la pureza y $0 \mathrm{Bx}$ de los materiales alimentados.

Bxl: Concentración inicial del material (\%)

Bx3: Concentración final de la masa cocida ( $\%)$

Bx2: Concentración del material alimentado (meladura o miel) $(\%)$

t: Tiempo de cocida de la templa $(\mathrm{h})$

STE: Sistema Termo energético

\section{Financiamiento}

Los autores no declaran haber recibido financiamiento para este artículo

\section{Conflicto de interés}

Los autores no declaran conflictos de interés que puedan afectar los resultados de este estudio.

\section{Bibliografía}

Alemán, L. F. M. 2017. «Evaluación energética y de la seguridad tecnológica ambiental en el central azucarero "Carlos Baliño"». (Trabajo de Diploma), Universidad Central "Marta Abreu" de Las Villas. Cuba.

Bernal, F. E. B. 2016. «Potencialidades de mejoras del esquema térmico del CAI 'Ciudad Caracas"». (Trabajo de Diploma). Universidad de Cienfuegos 'Carlos Rafael Rodríguez'. Cienfuegos, Cuba.

Díaz, L. D. V. 2016. «Oportunidades de mejoras del esquema térmico CAI 5 de septiembre» 
(Trabajo de Diploma), Universidad de Cienfuegos, Cuba.

Espinoza, R. 1991. Sistemas de utilización del calor en la industria azucarera. (ENPES ed.).

Falcón, M. G., Izquierdo, Y. G., Vicente, I. A., \& Suárez, E. G. 2019. «Evaluación del sistema energético en el central azucarero quintín bandera» Revista Centro Azúcar 46 (2019).

Hernández-Sardiñas, A., González Morales V.M, \& Freide Orozco, M.L. 2017. «Aprovechamiento de las posibles fuentes de biomasa para entregar más electricidad en la fábrica de azúcar Antonio Sánchez» Centro Azúcar 44 (4):88-97.

Hernández Touset, J.P, Espinosa Pedraja, R., Pérez Pérez, G., García Roque, A.M d.E \& García González, I.A. 2020. «Gestión energética en un central de azúcar crudo con uso del software sta 4.1» Gentro Azúcar 47 (1):77-89.

Iturralde, L.A, Jiménez Borges, R, Castro Perdomo, N.A, Monteagudo Yanes, J.P. 2020. «Metodología para balance energético de un Central azucarero. Estudio de caso: Central "Ciudad Caracas”» Revista Mapa 4 (20):27-47.

Jiménez Borges, R, Lorenzo Llanes, J, Monteagudo Yanes, J.P, Pérez de Alejo Victoria, H, Álvarez Delgado, R \& Carreño Sarmiento, D.D. 2017. «Potencialidades de entrega de energía eléctrica en dos centrales azucareros de la provincia de Cienfuegos» Centro Azúcar 44 (2):60-68.

Jiménez Borges, R, Lorenzo Llanes, J, Monteagudo Yanes, J.P. 2017. «Propuestas de mejora para incrementar la entrega de energía eléctrica en el Central Elpidio Gómez.» Universidad y Sociedad 9 (2):182-186.
Jiménez Borges, R, Monteagudo Yanes, J.P, Lorenzo Llanes, J. 2017. «Potencialidades de mejoras energéticas en el proceso tecnológico en los centrales azucareros.» Ingeniería Energética XXXVIII (2): 88-96.

Jiménez Borges, R. 2016. «Potencialidades de mejoras en el proceso tecnológico de los centrales azucareros de la provincia de Cienfuegos». (Tesis de Maestría) Universidad de Cienfuegos, Cuba.

Motito Legrá I, J.A, Soler Pérez, S.M \& Soto López, Y. 2013. «Estudio energético en la UEB Central Paquito Rosales de la provincia Santiago de Cuba». Tecnología Química XXXIV (1):7886.

Pérez, G. P. 2017. «Evaluación del sistema energético en la fábrica de azúcar Panchito Gómez Toro». (Trabajo de Diploma), Universidad Central "Marta Abreu" de Las Villas Cuba.

Pérez Ones, O, Gozá León, O. 2000. «Simulación y análisis del sistema termoenergético del CAI amistad con los pueblos. " Revista CubaAzúcar XXIX (4):15-19.

Rein, P. 2012. Ingeniería de la caña de azúcar. Berlín.

Victoria, H. E. P. d. A., González, A. P., Valdés, Y. G., Ones, O. P., Gómez, T. C., González, Y. G., Águila, R. M. 2009. «El análisis de procesos y el empleo adecuado de la energía en la producción de azúcar crudo y electricidad en ingenios cubanos». (Tesis Doctoral) Universidad de las Ciencias Informáticas, Instituto Superior Politécnico José Antonio Echeverría (ISPJAE). La Habana, Cuba. 